\title{
On the thermodynamics of quantum-electrodynamic frequency shifts
}

\author{
G W Ford $\dagger$, J T Lewis $\ddagger$ and R F O’Connell§ \\ $\dagger$ Department of Physics, The University of Michigan, Ann Arbor, Michigan 48109, USA \\ $\$$ School of Theoretical Physics, Dublin Institute for Advanced Studies, Dublin 4, Ireland \\ $\S$ Department of Physics and Astronomy, Louisiana State University, Baton Rouge, \\ Louisiana 70803 , USA
}

Received 29 October 1986

\begin{abstract}
A paper by Barton with the same title as this comments on some of our recent work in this general area and takes issue with some of our conclusions. Here, we point out that Barton's reinterpretation of his own previous work actually serves to support our conclusions. In particular, he is making extensive use of thermodynamic concepts (free energy, thermodynamic perturbation theory, ... ) which were introduced for the first time into this area in our papers.
\end{abstract}

\section{Introduction}

Once more, the interaction of atoms with black-body radiation is attracting much interest: in a paper with the same title as this one, Barton (1987) comments on some of our recent work in this area and restates the conclusions of his earlier paper (Barton 1972) in the light of our papers (Ford 1985, Ford et al 1985, 1986a, b).

First of all, we note that Barton agrees with our exact result for the oscillator; here our calculation depended crucially on-among other things-the inclusion of memory effects which take into account the dynamical character of the radiation field. Secondly, Barton now agrees with our contention that the frequencies which are observed spectroscopically are determined by differences in the Helmholtz free energies of atomic states. The main points which Barton seems to be making are (i) that our results are mathematically correct but that we have made unwarranted deductions, and (ii) that his method provides an alternative method for calculating the relevant difference in Helmholtz free energies; we agree with (ii) while rejecting (i). It would be unfortunate if his polemic were to obscure his elegant contribution to the subject.

Our approach is as follows:

(1) We consider an exactly soluble problem: the harmonic oscillator. The form of the solution enables us to draw conclusions which it would be impossible to draw from perturbation results.

(2) Using this exact solution as a guide, we discuss the atom case for which only perturbation results are available.

(3) The oscillator problem can be viewed as a special case of the atom problem, to which the atom results should apply.

(4) In particular: $(a)$ for $k T$ much greater than the level spacing we find a temperature-dependent free-energy shift which is independent of the oscillator potential. 
The same should be true for the atom; $(b)$ for $k T$ much less than the level spacing the shift is negligible. The same should be true for the atom.

For the benefit of the reader who does not wish to delve into all the details, we summarise, in $\S 2$, our results; in $\S 3$ we deal with some of the points raised by Barton.

\section{Description of our work}

To date, our published work on the subject of atomic systems interacting with blackbody radiation is contained in three joint publications (Ford et al 1985, 1986a, b). In addition, there is a brief conference proceedings report by one of us (Ford 1985). Since we feel these publications are complete and accurate as they stand, we shall here only briefly summarise the results, and try once again to explain our ideas.

The main point of Ford et al (1986a) is that an atom interacting with black-body radiation is a thermodynamic system. That is, the electromagnetic field acts as a thermal reservoir (heat-bath) at a temperature $T$ and the atom as a subsystem coupled to it. Now an essential feature of a reservoir is that it is a dynamical thing which can give and receive energy (heat). However, this exchange of heat is severely restricted by the second law of thermodynamics. It is for this reason that the concepts of thermodynamics (the distinction between heat and work, between work and energy, etc) are so useful as to be essential, in our opinion, for a discussion of the subject.

Once it is accepted that thermodynamic principles must be used in the atomic problem, it follows that the energy supplied by a photon (or photons) which drives a transition from, say, the ground state to an excited state is to be interpreted as the work done on the system. It is an established principle of thermodynamics (a special form, in fact, of the second law) that the work done (or, more generally, the minimum work done) in an isothermal transition is equal to the change in free energy (Landau and Lifshitz 1959). Therefore it is clear that the quantity of interest for comparison with experiment is the free energy $F(T)$ rather than the energy $U(T)$. Since the relation between the two is

$$
U=F-T \frac{\partial F}{\partial T}
$$

it is obvious that at zero of temperature, where most atomic effects are calculated, it makes no difference whether one uses $F$ or $U$, they are the same. However, when one considers finite temperature effects, the difference is important.

We have spoken of transitions between atomic states and the corresponding change in free energy. The point here is that an atom in a given state and interacting with black-body radiation is a metastable equilibrium state of the system. Thermodynamic concepts apply to metastable states. Indeed, the second law for irreversible processes deals with such states and, in particular, the principle that the work done in an isothermal transition equals the change in free energy applies to transitions between metastable states.

After these general remarks we now turn to the question of how we calculate these thermodynamic quantities. The energy of a system with Hamiltonian $H$ and in equilibrium at temperature $T$ is the thermal expectation of the Hamiltonian:

$$
U(T)=\operatorname{Tr}[H \exp (-H / k T)] / \operatorname{Tr}[\exp (-H / k T)] .
$$

The corresponding free energy is given by

$$
F(T)=-k T \ln \{\operatorname{Tr}[\exp (-H / k T)]\} .
$$


These formulae are so well known as to make any explanation unnecessary. They apply to an isolated system, i.e., a system whose interaction with the surroundings, once having established the temperature, may be neglected. An atom within a cavity filled with black-body radiation is such a system.

In Ford et al (1985) we obtained an exact expression for the free energy of an oscillator interacting via dipole coupling with black-body radiation. The Hamiltonian for this system is

$$
H=\frac{1}{2 m}\left(p_{x}-\frac{e}{c} A_{x}\right)^{2}+\frac{1}{2} K x^{2}+H_{\mathrm{R}}
$$

where the vector potential is

$$
A_{x}=\sum_{k, s}\left(\frac{2 \pi \hbar c}{k V}\right)^{1 / 2}\left(f_{k}^{*} a_{k, s} \hat{e}_{k, s} \cdot \hat{x}+f_{k} a_{k, s}^{+} \hat{e}_{k, s}^{*} \cdot \hat{x}\right)
$$

and the free radiation field Hamiltonian is

$$
H_{\mathrm{R}}=\sum_{k, s} \hbar c k a_{k, s}^{\dagger} a_{k, s} \text {. }
$$

The free energy ascribed to the oscillator is

$$
F_{0}(T)=F(T)-F_{\mathrm{R}}(T)
$$

where $F(T)$, given by (2.3) with $H$ given by (2.4), is the free energy of the interacting system and

$$
F_{\mathrm{R}}(T)=-k T \ln \left\{\operatorname{Tr}\left[\exp \left(-H_{\mathrm{R}} / k T\right)\right]\right\}=\frac{-\pi^{2} V(k T)^{4}}{45(\hbar c)^{3}}
$$

is the free energy of the black-body field in the absence of the oscillator. Thus $F_{0}(T)$ is the work done on the system when the oscillator is placed in the black-body field. In Ford et al (1985) we showed that this oscillator free energy is expressed by the remarkable formula,

$$
F_{0}=\frac{1}{\pi} \int_{0}^{\infty} \mathrm{d} \omega f(\omega, T) \operatorname{Im}\left(\frac{\mathrm{d} \ln (\alpha(\omega))}{\mathrm{d} \omega}\right)
$$

where

$$
f(\omega, T)=-k T \ln [1-\exp (-\hbar \omega / k T)]
$$

and where $\alpha(\omega)$ is the generalised susceptibility for the oscillator coupled to the radiation field. In Ford et al (1985) we obtained an expression for $\alpha(\omega)$ which explicitly involves a frequency $\Omega$, characterising the electron form factor, which in the end is to be taken very large. We have since discovered that this large- $\Omega$ limit can be taken first to obtain the following simple form:

$$
\alpha(\omega)=\frac{1-i \omega \gamma / \omega_{0}^{2}}{M\left(-\omega^{2}+\omega_{0}^{2}-i \omega \gamma\right)} .
$$

Here $M$ is the renormalised electron mass and

$$
\omega_{0}=(K / M)^{1 / 2} \quad \gamma=2 e^{2} \omega_{0}^{2} / 3 M c^{3} .
$$

With this (2.9) gives

$$
F_{0}(T)=\frac{1}{\pi} \int_{0}^{\infty} \mathrm{d} \omega f(\omega, T)\left(\frac{\gamma\left(\omega_{0}^{2}+\omega^{2}\right)}{\left(\omega_{0}^{2}-\omega^{2}\right)^{2}+\omega^{2} \gamma^{2}}-\frac{\gamma}{\omega_{0}^{2}+\omega^{2} \gamma^{2} / \omega_{0}^{2}}\right)
$$


In the high-temperature limit, where $k T \gg \hbar \omega_{0}$, but where $k T \ll \hbar \omega_{0}^{2} / \gamma=$ $3 M c^{3} \hbar / 2 e^{2}=105 \mathrm{MeV}$, this becomes

$$
F_{0}(T) \sim k T \ln \left(k T / \hbar \omega_{0}\right)+\frac{\pi e^{2}(k T)^{2}}{9 \hbar^{2} M c^{3}} \quad k T \gg \hbar \omega_{0} .
$$

The corresponding energy, using $(2.1)$, is

$$
U_{0}(T) \sim k T-\frac{\pi e^{2}(k T)^{2}}{9 \hbar M c^{3}} \quad k T \gg \hbar \omega_{0} .
$$

Here the first term is the familiar equipartition energy of the high-temperature oscillator. The second term, which, in practice, is always small compared with the first, is a quantum-electrodynamic correction, corresponding to a shift in the energy relative to its equipartition value. We stress that this shift is negative. The corresponding shift of the free energy, however, is positive.

In the low-temperature limit, $k T \ll \hbar \omega_{0}$, we find

$$
\begin{array}{ll}
F_{0}(T) \sim-\frac{2 \pi^{3} e^{2}(k T)^{4}}{45 M \omega_{0}^{2}(\hbar c)^{3}}\left[1-\frac{1}{3}\left(\frac{2 e^{2} \omega_{0}}{3 M c^{3}}\right)^{2}\right] & k T \ll \hbar \omega_{0} \\
U_{0}(T) \sim \frac{2 \pi e^{2}(k T)^{4}}{15 M \omega_{0}^{2}(\hbar c)^{3}}\left[1-\frac{1}{3}\left(\frac{2 e^{2} \omega_{0}}{3 M c^{3}}\right)^{2}\right] & k T \ll \hbar \omega_{0} .
\end{array}
$$

In general this energy in the low-temperature limit is very small.

We turn now to the physical interpretation of the terms in our expression (2.9) for the oscillator free energy. Our ultimate aim is to identify the free-energy shifts to be attributed to the metastable states corresponding to the individual oscillator levels. We can do this because ours is an exact, non-perturbative, result whose form allows the identification.

What we do is to consider a comparison system, a thermodynamic system whose properties one can recognise and the expression for whose free energy, when compared with that of the given system, allows one to identify the physical meaning of the additional terms. In our case the comparison system is an oscillator coupled with what we might call a Drude heat-bath. This is a heat-bath which gives rise to a frequencyindependent friction constant, i.e., a Drude model in which the radiation reaction force is proportional to the instantaneous velocity. The FKM model is a microscopic model of such a heat-bath (Ford et al 1965). A much earlier and physically more transparent microscopic model is that of Horace Lamb (Lamb 1900, Lewis and Thomas 1974, Lewis and Maassen 1984) who showed that a particle attached to a stretched string has a frequency-independent friction constant. Now the first term in (2.13)

$$
F_{\mathrm{OD}}(T)=\frac{1}{\pi} \int_{0}^{\infty} \mathrm{d} \omega f(\omega, T) \frac{\gamma\left(\omega^{2}+\omega_{0}^{2}\right)}{\left(\omega^{2}-\omega_{0}^{2}\right)^{2}+\omega^{2} \gamma^{2}}
$$

is the free energy of an oscillator interacting with a Drude heat-bath; the friction constant being $M \gamma$. We know, moreover, that for a Drude heat-bath the energy of interaction is zero. This is seen most simply in the Lamb model, since the requirement of attachment is simply a mechanical constraint, with no associated potential energy of interaction (Lewis and Maassen 1984). We know, therefore, that for a Drude bath there are no shifts of the oscillator levels, only a broadening due to the finite lifetime 
of the states. We conclude that (2.16) represents the free energy associated with the broadened unshifted oscillator levels, and that in (2.13) the additional term

$$
\Delta F_{0}(T)=-\frac{\gamma}{\pi \omega_{0}^{2}} \int_{0}^{\infty} \mathrm{d} \omega f(\omega, T)=\frac{\pi e^{2}(k T)^{2}}{9 \hbar M c^{3}}
$$

is a uniform shift in free energy of the system in each level.

In Ford et al (1986a) we used our exact results for the oscillator to discuss the case of the Rydberg atom. The point here is that the free-energy shift (2.19) is independent of the oscillator force constant and, in the high-temperature limit, is essentially that for a free electron. It should therefore apply to the closely spaced high Rydberg levels of an atom. Note that we only claim the result (2.19) applies to the Rydberg levels in the high-temperature limit, where $k T$ is large compared with the level spacing. For the tightly bound lower levels, whose spacing is large compared with $k T$, we can infer from the low-temperature result $(2.16)$ that the shift will be small compared with that for the upper levels. It would follow that the shift observed in the photon absorption spectrum corresponds to the shift in free energy of the upper levels alone, given by (2.19).

Finally, as we announced in Ford et al (1985), in Ford et al (1986b) we applied thermodynamic perturbation theory to the problem of an atom interacting with blackbody radiation. The result is that the temperature-dependent part of the free energy of interaction can be written in the form

$$
\delta \tilde{F}(T)=\sum_{a} \frac{\exp \left(-\varepsilon_{a} / k T\right)}{Z} \delta f_{a}(T)
$$

where the sum is over atomic levels with energy $\varepsilon_{a}, Z=\Sigma_{a} \exp \left(-\varepsilon_{a} / k T\right)$, and

$$
\delta f_{a}(T)=\frac{e^{2} \hbar^{2}}{3 \pi^{2} m^{2}} \int \mathrm{d} k \frac{\hbar \omega}{\exp (\hbar \omega / k T)-1} \sum_{b} \frac{\left|\left\langle\phi_{a}, p \phi_{b}\right\rangle\right|^{2}}{\varepsilon_{a}-\varepsilon_{b}} \frac{1}{\left(\varepsilon_{a}-\varepsilon_{b}\right)^{2}-\hbar^{2} \omega^{2}} .
$$

Note that only the bare mass $m$ appears; the mass renormalisation, while divergent, is of higher order. In the high-temperature limit we use the $f$ sum rule to get the general result:

$$
\delta f_{a}(T) \sim \frac{\pi e^{2}(k T)^{2}}{3 \hbar m c^{3}} \quad k T \gg\left|\varepsilon_{a}-\varepsilon_{b}\right| .
$$

The factor three difference from (2.19) arises from the three dimensions of space. This high-temperature limit is independent of the atomic state and we show that it is to be interpreted as a shift in the free energy of each state, not the energy. Thus, the results of time-independent perturbation theory confirm our previous results.

\section{Relation with other work}

In many applications of thermodynamics, when one wishes to discuss non-equilibrium states, one can envisage these to be states of constrained equilibrium. In the language of statistical mechanics this means one adjusts certain parameters in the Hamiltonian so that the state is an equilibrium state of the adjusted Hamiltonian and one can then use (2.3) to form the free energy. It is this free energy which enters, e.g., in the 
calculation of the change of free energy in a transition to the equilibrium state. However, for an atom interacting with black-body radiation this is not possible since the same interaction terms which bring about the metastable equilibrium of an excited state are responsible for the decay of the state. It is for this reason that we resorted to the indirect method of the comparison system described in $\S 2$. What Barton does when faced with the same difficulty is to write down ad hoc a prescription for the free energy of the excited state.

As an example of the sort of error which still seems to be present in the discussion of Barton, we consider the familiar AC Stark shift in relation to our results. When an atom is placed in a very high-frequency classical electric field $E_{0} \cos (\omega t)$ it acquires an additional vibrational kinetic energy

$$
W(\omega)=\frac{e^{2} E_{0}^{2}}{4 m \omega^{2}} .
$$

In this same field the AC Stark shift is given by the formula (Townes and Schawlow 1955)

$$
\delta \varepsilon_{a}=\frac{e^{2} E_{0}^{2}}{4} \sum_{b}\left|\left\langle\phi_{a}, x \phi_{b}\right\rangle\right|^{2}\left(\frac{1}{\varepsilon_{a}-\varepsilon_{b}-\hbar \omega}+\frac{1}{\varepsilon_{a}-\varepsilon_{b}+\hbar \omega}\right) .
$$

In the high-frequency limit, using the $f$ sum rule (Bethe and Salpeter 1957),

$$
\sum_{b}\left(\varepsilon_{a}-\varepsilon_{b}\right)\left|\left\langle\phi_{a}, x \phi_{b}\right\rangle\right|^{2}=-\hbar^{2} / 2 m
$$

this becomes identical with (3.1), independent of the atomic state. Thus the AC Stark shift, in the high-frequency limit, is just the vibrational kinetic energy. We should emphasise that these formulae, and their derivation in Townes and Schawlow (1955), are perfectly correct.

In adapting these formulae to the case of black-body radiation what has generally been done is to replace $3 E_{0}^{2} / 8$ with the Planck energy density

$$
\rho(\omega, T)=\left(\hbar \omega^{3} / \pi^{2} c^{3}\right) /[\exp (\hbar \omega / k T)-1]
$$

Integrating over $\omega$ one finds for the mean vibrational energy

$$
\bar{W}=\int_{0}^{\infty} \mathrm{d} \omega W(\omega)=\frac{\pi e^{2}(k T)^{2}}{9 \hbar m c^{3}} .
$$

This is identical with the high-temperature shift in the free energy $F_{0}(T)$, given by (2.17). However, $\bar{W}$ is an energy and, for a $T^{2}$ temperature dependence, energy and free energy have opposite signs.

To explain this contradiction, we consider the mean kinetic energy of the oscillator within our formalism. This we define to be

$$
\left\langle: \frac{1}{2} M \dot{x}^{2}:\right\rangle=\operatorname{Tr}\left[: \frac{1}{2} M \dot{x}^{2}: \exp (-H / k T)\right] / \operatorname{Tr}[\exp (-H / k T)]
$$

where we use the normal product since we are interested only in the temperaturedependent effects. This, in turn, can be expressed in terms of the generalised susceptibility $\alpha(\omega)$ through the fluctuation-dissipation theorem (Landau and Lifshitz 1959):

$$
\left\langle: \frac{1}{2} M \dot{x}^{2}:\right\rangle=\frac{M \hbar}{\pi} \int_{0}^{\infty} \mathrm{d} \omega \frac{\omega^{2} \operatorname{Im}(\alpha(\omega))}{\exp (\hbar \omega / k T)-1} .
$$

Using the expression (2.11) for $\alpha$ this becomes

$$
\left\langle: \frac{1}{2} M \dot{x}^{2}:\right\rangle=\frac{\hbar \gamma}{\pi \omega_{0}^{2}} \int_{0}^{\infty} \mathrm{d} \omega \frac{\omega^{5}}{\left[\left(\omega_{0}^{2}-\omega^{2}\right)^{2}+\omega^{2} \gamma^{2}\right][\exp (\hbar \omega / k T)-1]} .
$$


In the high-temperature limit this becomes

$$
\left\langle: \frac{1}{2} M \dot{x}^{2}:\right\rangle \sim \frac{1}{2} k T\left(1-\frac{\gamma^{2}}{\omega_{0}^{2}}\right)+\frac{\pi \gamma(k T)^{2}}{6 \hbar \omega_{0}^{2}} .
$$

Here the first term is the equipartition kinetic energy, with a very small correction due to finite level width, while the second term, using (2.12), is identical with (3.5). Thus we see that the above heuristic argument, in fact, correctly gives the positive $T^{2}$ correction to the vibrational kinetic energy at high temperature.

How then do we understand the apparent contradiction with our exact result, which gives a negative $T^{2}$ correction to the oscillator energy? As we have defined it, this oscillator energy is the energy of the black-body cavity containing the oscillator minus the energy of the same cavity without the oscillator. This is an operational definition corresponding with the (ideally) measured quantity. However, the kinetic energy is only a part of this oscillator energy, there is the energy of interaction with the field and the change in field energy due to the presence of the oscillator. Indeed, the renormalised mass $M$ is almost entirely of electromagnetic origin.

We find it necessary to refute directly three of Barton's statements.

(a) Barton claims that '. . the harmonic oscillator ... is so special a case as to be totally unrepresentative ... of any atomic system'. As we stressed in Ford et al (1986a), this is not correct; the free-energy shift for the oscillator is independent of the oscillator force constant and thus it applies to any nearly-free electron; in particular, it applies to an electron in a high Rydberg level of an atom.

(b) Barton remarks that in the oscillator, the unconstrained and all the constrained free-energy shifts happen to coincide and, consequently, it suffers no spectroscopically measurable thermal frequency shift at all'-a statement with which we are in total agreement-but he then goes on to say that '... FLO give no inkling of this'. This is not so; in Ford et al (1985) we state that ' $\ldots F_{0}(T)$ is to be interpreted as a temperaturedependent shift in free-energy of each level'.

(c) Finally, Barton-in discussing the experiment reported by Hollberg and Hall (1984)-remarks that ' $\ldots$ an acceptable explanation must account for the difference between ... free-energy shifts of the upper and lower levels, which FLO's theory does not address at all'. Again, this is simply incorrect. The Hollberg-Hall experiment utilises a tightly-bound initial state of the electron and a high Rydberg level for the final state of the electron. We concerned ourselves only with the high Rydberg level since, as is well known, the shift in the free-energy of the tightly-bound lower level is negligible.

In conclusion, we believe that Barton (1987) gives support to our introduction of thermodynamic concepts into the discussion of problems concerning the effects of black-body radiation on atomic phenomena. We accept that the calculations in Barton (1972) can be re-interpreted as calculations of the shift in the Helmholtz free energy; however, the only clues to this are provided by the revised description of these calculations presented in Barton (1987).

\section{Acknowledgment}

This research was partially supported by the US Office of Naval Research under contract No N00014-86-K-0002 and by the National Science Foundation, grant No INT-8504402. 


\section{References}

Barton G G 1972 Phys. Rev. A 5468

1987 J. Phys. B: At. Mol. Phys. 20 879-98

Bethe H A and Salpeter E E 1957 Quantum Mechanics of One- and Two-Electron Atoms (Berlin: Springer) $\S 62$

Ford G W 1985 Lecture Notes in Mathematics No 1136 (Heidelberg: Springer) p 202

Ford G W, Kac M and Mazur P 1965 J. Math. Phys. 6504

Ford G W, Lewis J T and O'Connell R F 1985 Phys. Rev. Lett. 552273

- 1986a J. Phys. B: At. Mol. Phys. 19 L41

1986b Phys. Rev. A 343001

Hollberg L and Hall J L 1984 Phys. Rev. Lett. $\mathbf{5 3} 230$

Lamb H 1900 Proc. Lond. Math. Soc. 288

Landau L D and Lifshitz E M 1959 Statistical Physics 3rd edn (London: Pergamon)

Lewis J T and Maassen H 1984 Quantum Probability and Applications ed L Accardi, A Frigerio and V Gorini, Lecture Notes in Mathematics No 1055 (Heidelberg: Springer)

Lewis J T and Thomas L C 1974 Functional Integration ed A M Arthurs (Oxford: Clarendon)

Townes C F and Schawlow A L 1955 Microwave Spectroscopy (New York: McGraw-Hill) 